\title{
Prevalence of possible idiopathic normal pressure hydrocephalus in older inpatients with schizophrenia: a replication study
}

Yuta Yoshino ${ }^{1}$, Taku Yoshida ${ }^{1}$, Hideo Morino ${ }^{2}$, Masayuki Nakamura ${ }^{3}$, Masao Abe ${ }^{1}$, Hokuto Omachi ${ }^{1}$, Saori Inoue ${ }^{1}$, Yukiyo Miyoshi ${ }^{1}$, Yumina Tachibana ${ }^{1}$, Noriko Yamauchi ${ }^{2}$, Naoya Takeda ${ }^{3}$, Mutsuhiko Mizobuchi ${ }^{4}$, Yuki Ozaki ${ }^{1}$, Shinichiro Ochi ${ }^{1}$, Junichi lga ${ }^{1 *}$ (iD and Shu-ichi Ueno ${ }^{1}$

\begin{abstract}
Background: We recently reported that older patients with schizophrenia (SZ) show possible idiopathic normal pressure hydrocephalus (iNPH) more frequently than the general population. In this study, we estimated the prevalence of $\mathrm{NPH}$ in a larger number of older SZ patients and explored useful examination values for diagnosis in the SZ population.

Methods: We enrolled older inpatients with $S Z(n=39$, mean age $=68.6 \pm 7.7$ years) from several psychiatric hospitals in Ehime, Japan and acquired brain imaging data using computed tomography. We evaluated three iNPH symptoms (dementia, gait disturbance, and urinary incontinence). In addition, we combined these data with our previous data to elucidate the relationship between iNPH and characteristics of SZ symptoms.

Results: In total, five (12.8\%) patients were diagnosed with possible iNPH. Evans' index for patients with iNPH was significantly higher than for those without iNPH ( $p=0.002)$. The number of disproportionately enlarged subarachnoid space hydrocephalus (DESH) findings was significantly higher in patients with iNPH than in those without iNPH $(p<0.001)$. Using combined data, Drug-Induced Extra-pyramidal Symptoms Scale (DIEPSS) subscales of gait and bradykinesia showed an increasing trend in the SZ with iNPH group.
\end{abstract}

Conclusions: We reconfirmed that older inpatients with SZ experienced possible iNPH more frequently than the general population. We should pay attention to the DIEPSS subscales of gait and bradykinesia and DESH findings in addition to the three main symptoms of iNPH and Evans' index so as to not miss SZ patients with iNPH.

Keywords: Schizophrenia, Normal pressure hydrocephalus, Cerebrospinal fluid tap test, Disproportionately enlarged subarachnoid space, Drug-induced extra-pyramidal symptoms scale

\footnotetext{
* Correspondence: igajunichi@hotmail.com

${ }^{1}$ Department of Neuropsychiatry, Molecules and Function, Ehime University Graduate School of Medicine, Shitsukawa, Toon, Ehime 791-0295, Japan

Full list of author information is available at the end of the article
}

C C The Author(s). 2020 Open Access This article is licensed under a Creative Commons Attribution 4.0 International License, which permits use, sharing, adaptation, distribution and reproduction in any medium or format, as long as you give appropriate credit to the original author(s) and the source, provide a link to the Creative Commons licence, and indicate if changes were made. The images or other third party material in this article are included in the article's Creative Commons. licence, unless indicated otherwise in a credit line to the material. If material is not included in the article's Creative Commons licence and your intended use is not permitted by statutory regulation or exceeds the permitted use, you will need to obtain permission directly from the copyright holder. To view a copy of this licence, visit http://creativecommons.org/licenses/by/4.0/. The Creative Commons Public Domain Dedication waiver (http://creativecommons.org/publicdomain/zero/1.0/) applies to the data made available in this article, unless otherwise stated in a credit line to the data. 


\section{Background}

The prevalence of physical comorbidities is higher in patients with schizophrenia (SZ) compared to the general population [1]. Common and serious comorbidities include metabolic syndrome [2] caused by secondgeneration antipsychotics [3] and/or cognitive impairment that leads to problems with adherence to those treatments [4]. To our knowledge, only limited studies have mentioned idiopathic normal pressure hydrocephalus (iNPH) among SZ patients, including one retrospective study [5], two case reports [6, 7], and one theoretical paper [8].

iNPH is treatable dementia with three main symptoms, which are dementia (psychomotor slowing and impaired attention, executive and visuospatial dysfunction), gait disturbance (shuffling, magnetic, and wide-based), and urinary incontinence [9, 10]. As for SZ symptoms, SZ patients also show cognitive impairments including psychomotor slowing, impaired attention, and executive function $[11,12]$. Furthermore, antipsychotics often induce gait disturbance with Parkinsonism. Distinguishing iNPH symptoms from SZ symptoms or side effects of antipsychotics can be difficult, and it is possible to miss the iNPH symptoms in the daily clinical situations.

Surgical placement of a shunt is the only way to improve iNPH symptoms and prevent cerebrospinal fluid (CSF) storage. The CSF tap test is a valid method for diagnosis and for prediction of response to shunting [13-15]. Picascia et al. [16] suggested that shunt surgery in the early stage of iNPH may prevent progression of motor disturbances and cognitive impairment. In addition, adequate shunt surgery may improve the quality of life of iNPH patients [17].

The pathogenesis of iNPH is still unclear but there are considerable hypotheses based on previous evidence as Brautigam et al. reviewed [18]. There are four considerable etiologic and physiopathologic points (abnormal cerebrospinal fluid dynamics, vascular etiology, inflammation, and hereditary factors). As result of those, ventriculomegaly is consistent in iNPH patients. However, the ventriculomegaly can be seen in schizophrenia patients due to the increased volume of ventricles especially in elderly SZ patients [19]. Additionally, it is reported that the long-term medication of antipsychotics and benzodiazepine is associated with these brain structural changes [20]. The increase ventricles volume in SZ patients possibly mask the ventriculomegaly due to iNPH. In terms of risk factors for iNPH, diabetes mellitus (DM) is one of the possible risk factors, which can be involved for a vascular etiology [21, 22]. The risk of type $2 \mathrm{DM}$ is 2 - to 5 -fold higher in SZ patients compared to general population [23]. In addition, it has been reported that metabolic syndrome itself (DM, hypertension, and hyperlipidemia) are also risk factors for iNPH
[24-26]. We have hypothesized that these reasons increase the prevalence of iNPH in SZ population compared with the general population, and that is also higher the previously estimated prevalence in SZ population (3.1\%).

In our preceding study, we reported the possibility of a higher prevalence (14.3\%) of iNPH in Japanese SZ patients compared to the general population (0.51\%) [27, 28 ]. However, the sample size of this study was relatively small $(n=21)$ and involved patients in a single hospital. In our current study, we recruited a larger number of older SZ inpatients from several hospitals and assessed both iNPH and SZ symptoms to reveal the prevalence of iNPH in SZ patients. Moreover, we combined these data with our preceding data to elucidate the relationship between iNPH and the characteristic SZ symptoms.

\section{Methods}

\section{Subjects}

We enrolled 39 SZ patients who were hospitalized in Matsukaze Hospital, Juzen Yurinoki Hospital, or Shokokai Imabari Hospital in Ehime, Japan from November 2017 to March 2018. SZ was diagnosed according to Diagnosis and Statistical Manual of Mental Disorder (DSM-5) criteria by at least two expert psychiatrists based on extensive clinical interviews and a review of medical records. The diagnosis of possible iNPH was conducted using the criteria proposed by the guidelines [29]. In detail, the required criteria are as follows: 1) Symptoms occur in the 60s or older, 2) More than one of the following clinical symptoms: gait disturbance, cognitive impairment, and urinary incontinence, 3) Ventricular dilation (Evans' index >0.3), 4) Clinical symptoms of iNPH cannot be completely explained by other neurological or non-neurological diseases, and 5) Preceding diseases possibly causing ventricular dilation are not obvious. The patients with a history of brain injury or stroke and those who used wheelchairs were excluded. All subjects were of unrelated Japanese origin and signed written informed consent forms approved by the institutional ethics committees of Matsukaze Hospital, Juzen Yurinoki Hospital, Shokokai Imabari Hospital, and Ehime University Graduate School of Medicine.

\section{Assessment of symptoms of SZ and iNPH}

Symptoms of SZ were assessed by the Positive and Negative Symptom Scale (PANSS) (each item is scored on a 1-7 scale) [30]. For the combined data analysis, the Brief Psychiatric Rating Scale (BPRS) score was estimated by PANSS according to a previous paper [31]. We evaluated antipsychotic-induced extrapyramidal symptoms using the Drug-Induced Extrapyramidal Symptoms Scale (DIEPSS) [32]. 
We evaluated three major iNPH symptoms including gait disturbance, cognitive disturbance, and urinary disturbance with the same tests as in our preceding study [28]. The iNPH grading scale (iNPHGS) included three items: gait disturbance (GS-Gait), cognitive impairment (GS-Cognition), and urinary incontinence (GS-Urine). Patients and caregivers were interviewed to assess iNPHGS [33].

Timed Up and Go (TUG) [34] and the 10-m walking test [35] were used to assess gait disturbances. We conducted those tests twice, and the mean scores were calculated. Gait disturbances were also assessed by the Gait Status Scale (GSS) [33].

Cognitive impairment was evaluated with the MiniMental State Examination (MMSE) [36]. Caregivers (the attending nurse in this study) assessed neuropsychiatric symptoms with the neuropsychiatric inventory (NPI) [37].

Imaging data were obtained using a computed tomography (CT) scanner. We defined results for the Evans' index $>0.3$ as abnormal [21].

\section{Genotyping}

Genotyping of single-nucleotide polymorphisms (rs429358 and rs7412) of apolipoprotein $\mathrm{E}(A P O E)$ was conducted using the TaqMan $5^{\prime}$-exonuclease allelic discrimination assay (Assay ID: rs429538; C__3084793_20 and rs7412, respectively, Applied Biosystems) using the StepOnePlus realtime PCR system (Applied Biosystems). Genotyping call rates were 100.0\% (rs429358) and 100.0\% (rs7412). We found no deviation from the Hardy-Weinberg equilibrium in each examined single-nucleotide polymorphism in the patients $(p>0.05)$. The APOE isotype-related genotypes are combinations of the $A P O E \varepsilon 2, \varepsilon 3$, and $\varepsilon 4$ alleles derived from the two genotypes of rs429358 (T334C) and rs7412 (C472T): $\varepsilon 2,334 \mathrm{~T} / 472 \mathrm{~T}$; $\varepsilon 3,334 \mathrm{~T} / 472 \mathrm{C}$; and $\varepsilon 4,334 \mathrm{C} / 472 \mathrm{C}$. The $\varepsilon 4$ genotype is a risk factor for Alzheimer's disease (AD) [38].

\section{Statistical analysis}

Statistical analyses were conducted with EZR version 1.36 [39]. The Shapiro-Wilk test was used to test for normality. Comparisons between no iNPH and possible iNPH patients were conducted for age, body mass index (BMI), onset of age, duration of illness, chlorpromazine (CP) equivalent dose, PANSS, DIEPSS, iNPHGS, TUG, 10-m walking test, GSS, MMSE, NPI, and Evans' index. Student's $t$-test was used for normally distributed data, and the Mann-Whitney $U$ test was used for those not normally distributed. The average DIEPSS subscales were analyzed with Student's $t$-test or the MannWhitney $U$ test with Bonferroni correction. The differences in sex, smoking status, hypertension, hyperlipidemia, diabetes mellitus, disproportionately enlarged subarachnoid space hydrocephalus (DESH), and APOE genotypes were analyzed using the Fisher's exact test. Correlations for each parameter were analyzed by determining the Pearson's correlation coefficient. Statistical significance was defined at the 95\% level $(p=0.05)$.

\section{Results}

\section{No iNPH vs. possible iNPH groups}

All the possible samples were recruited except samples meeting exclusion criteria or who refused this study. Finally, 38 subjects were collected, and those characteristics and results of their examinations are shown in Table 1. In total, five of 38 (12.8\%) patients were diagnosed with possible iNPH. We excluded one patient with secondary NPH from statistical analysis. In this case, NPH was thought to be caused by a mass that was detected with CT (Fig. 1f). Evans' index in patients with iNPH was significantly higher than in those without iNPH $(p=0.002)$. The number of DESH findings was significantly higher in patients with iNPH than in those without iNPH $(p<0.001)$.

Gender $(p=0.643)$, age $(p=0.352)$, BMI $(p=0.133)$, smoking status $(p=0.555)$, hypertension $(p=1.0)$, hyperlipidemia $(p=1.0)$, diabetes mellitus $(p=1.0)$, age of onset $(p=0.632)$, duration of illness $(p=0.436), \mathrm{CP}$ equivalent $(p=0.863)$, use of anticholinergics $(p=1.0)$, use of benzodiazepine $(p=0.342)$, use of mood stabilizers $(p=0.342)$, PANSS-total $(p=0.931), \quad$ PANSS-positive $(p=0.363)$, PANSS-negative $(p=0.914)$, PANSS-general $(p=0.762)$, DIEPSS $(p=0.062)$, iNPHGS-Gait $(p=0.082)$, iNPHGSCogn $(p=0.471)$, iNPHGS-Urin $(p=0.425)$, TUG $(p=$ $0.267), 10-\mathrm{m}$ walking test $(p=0.858)$, GSS $(p=0.192)$, MMSE $(p=0.281)$, NPI $(p=0.160)$, and APOE genotype $(p=1.0)$ were not significantly different (Table 1$)$ between groups. CT data of possible iNPH and secondary NPH patients are shown in Fig. 1.

\section{Analysis of combined data from this and the previous study}

The demographics of the combined data and the results of statistical analysis are shown in Table 2. Eight of 59 (13.6\%) SZ patients were diagnosed with possible iNPH as a comorbidity. The average DIEPSS $(p=0.006)$, iNPHGS-Gait $(p=0.015)$, GSS $(p=0.009)$, and Evans' index $(p<0.001)$ were significantly higher in patients with possible iNPH compared to patients without iNPH. The proportion of patients with DESH was higher in the possible iNPH group than in the no iNPH group (4/51 vs. $5 / 8, p=0.001$ ).

We found no significant differences in sex $(p=1.0)$, age $(p=0.092)$, BMI $(p=0.294)$, smoking $(p=0.176)$, age of onset $(p=0.912)$, duration of illness $(p=0.069)$, CP equivalent $(p=0.842)$, use of anticholinergics $(p=0.698)$, BPRS $(p=0.765)$, iNPHGS-Cogn $(p=0.107)$, iNPHGSUrin $(p=0.178)$, TUG $(p=0.376), 10-\mathrm{m}$ walking test 
Table 1 Demographic and clinical data of patients with possible iNPH and without iNPH (no iNPH)

\begin{tabular}{|c|c|c|c|}
\hline Characteristics & No iNPH $(n=33)$ & Possible iNPH $(n=5)$ & $p$ value \\
\hline Sex, male & 21 & 4 & 0.643 \\
\hline Age (years) & $68.3 \pm 7.4$ & $72.2 \pm 9.6$ & 0.352 \\
\hline $\mathrm{BMI}\left(\mathrm{kg} / \mathrm{m}^{2}\right)$ & $21.5 \pm 2.3(29)$ & $23.4 \pm 2.7(4)$ & 0.133 \\
\hline Smoking & 9 & 0 & 0.555 \\
\hline Hypertension & 7 & 1 & 1.0 \\
\hline Hyperlipidemia & 9 & 1 & 1.0 \\
\hline Diabetes Mellitus & 1 & 0 & 1.0 \\
\hline Age of onset (years) & $23.2 \pm 8.1$ & $24.8 \pm 7.3$ & 0.632 \\
\hline Duration of illness (months) & $45.0 \pm 7.9$ & $47.4 \pm 13.2$ & 0.436 \\
\hline $\mathrm{CP}$ equivalent & $820.9 \pm 500.9$ & $741.2 \pm 591.4$ & 0.863 \\
\hline \multicolumn{4}{|l|}{ Drug administration } \\
\hline Anticholinergics & 10 & 2 & 1.0 \\
\hline Benzodiazepines & 23 & 2 & 0.342 \\
\hline Antidepressants & 0 & 0 & - \\
\hline Mood stabilizers & 13 & 0 & 0.342 \\
\hline PANSS-total & $89.4 \pm 15.4$ & $87.0 \pm 18.9$ & 0.931 \\
\hline PANSS-positive & $21.6 \pm 5.1$ & $18.4 \pm 5.7$ & 0.363 \\
\hline PANSS-negative & $24.3 \pm 6.9$ & $24.8 \pm 7.0$ & 0.914 \\
\hline PANSS-general & $43.5 \pm 9.2$ & $43.8 \pm 6.7$ & 0.762 \\
\hline DIEPSS & $5.8 \pm 5.0$ & $10.4 \pm 5.0$ & 0.062 \\
\hline \multicolumn{4}{|l|}{ iNPHGS } \\
\hline GS-Gait & $0.91 \pm 1.2$ & $2.2 \pm 1.8$ & 0.082 \\
\hline GS-Cogn & $1.9 \pm 1.2$ & $2.4 \pm 1.5$ & 0.471 \\
\hline GS-Urin & $1.0 \pm 1.6$ & $1.6 \pm 1.8$ & 0.425 \\
\hline TUG & $14.7 \pm 6.6(26)$ & $10.6 \pm 2.7(3)$ & 0.267 \\
\hline 10-m walking test & $14.0 \pm 4.9(26)$ & $15.3 \pm 7.3(3)$ & 0.858 \\
\hline GSS & $3.2 \pm 3.1(29)$ & $5.0 \pm 2.6(3)$ & 0.192 \\
\hline MMSE & $19.6 \pm 6.3(31)$ & $15.6 \pm 8.3$ & 0.281 \\
\hline NPI & $15.4 \pm 11.4$ & $8.0 \pm 7.9$ & 0.160 \\
\hline Evans' Index & $0.27 \pm 0.04$ & $0.34 \pm 0.03$ & 0.002 \\
\hline DESH & 2 & 4 & $<0.001$ \\
\hline APOE $\varepsilon 4+/ \varepsilon 4-$ & $8 / 25$ & $1 / 4$ & 1.0 \\
\hline
\end{tabular}

Values denote number (\%) or mean \pm standard deviation

$B M I$ body mass index, CP equivalent chlorpromazine equivalent, DESH disproportionately enlarged subarachnoid space hydrocephalus, DIEPSS Drug-Induced Extrapyramidal Symptoms Scale, iNPH idiopathic normal pressure hydrocephalus, iNPHGS idiopathic Normal-Pressure Hydrocephalus Grading Scale, GS-Gait iNPHGS for gait, GS-Cogn iNPHGS for cognition, GS-Urin iNPHGS for urinary function, TUG Timed Up-and-Go test, GSS Gait Status Scale, MMSE Mini-Mental State Examination PANSS Positive and Negative Syndrome Scale, NPI neuropsychiatric inventory

$(p=0.064)$, MMSE $(p=0.317)$, NPI $(p=0.451)$, or $A P O E$ genotype $(p=1.0)$ between groups.

Subsequently, we analyzed the subscales of DIEPSS (Table 3). None of the subscales were significantly different between groups after Bonferroni correction (Gait: $p=$ 0.021; Bradykinesia: $p=0.015$; Sialorrhea: $p=0.531$; Rigidity: $p=0.063$; Tremor: $p=0.376$; Akathisia: $p=0.021$; Dystonia: $p=0.145$; Dyskinesia: $p=0.468$; Global: $p=0.011$ ).

None of the MMSE subscales were significantly different between groups after Bonferroni correction (Orientation in time: $p=0.012$; Orientation in place: $p=0.968$; Registration: $p=0.048$; Serial-7: $p=0.124$; Repetition: $p=0.189$; Three-stage command: $p=0.724$; Reading: $p=0.179$; Recall: $p=0.443$; Naming: $p=0.023$; Writing: $p=0.179$; Construction: $p=0.423$, Table 4 ).

\section{Discussion}

This study has three major findings.

First, we found that five of 38 (12.8\%) subjects had possible iNPH, which is similar to our preceding study 


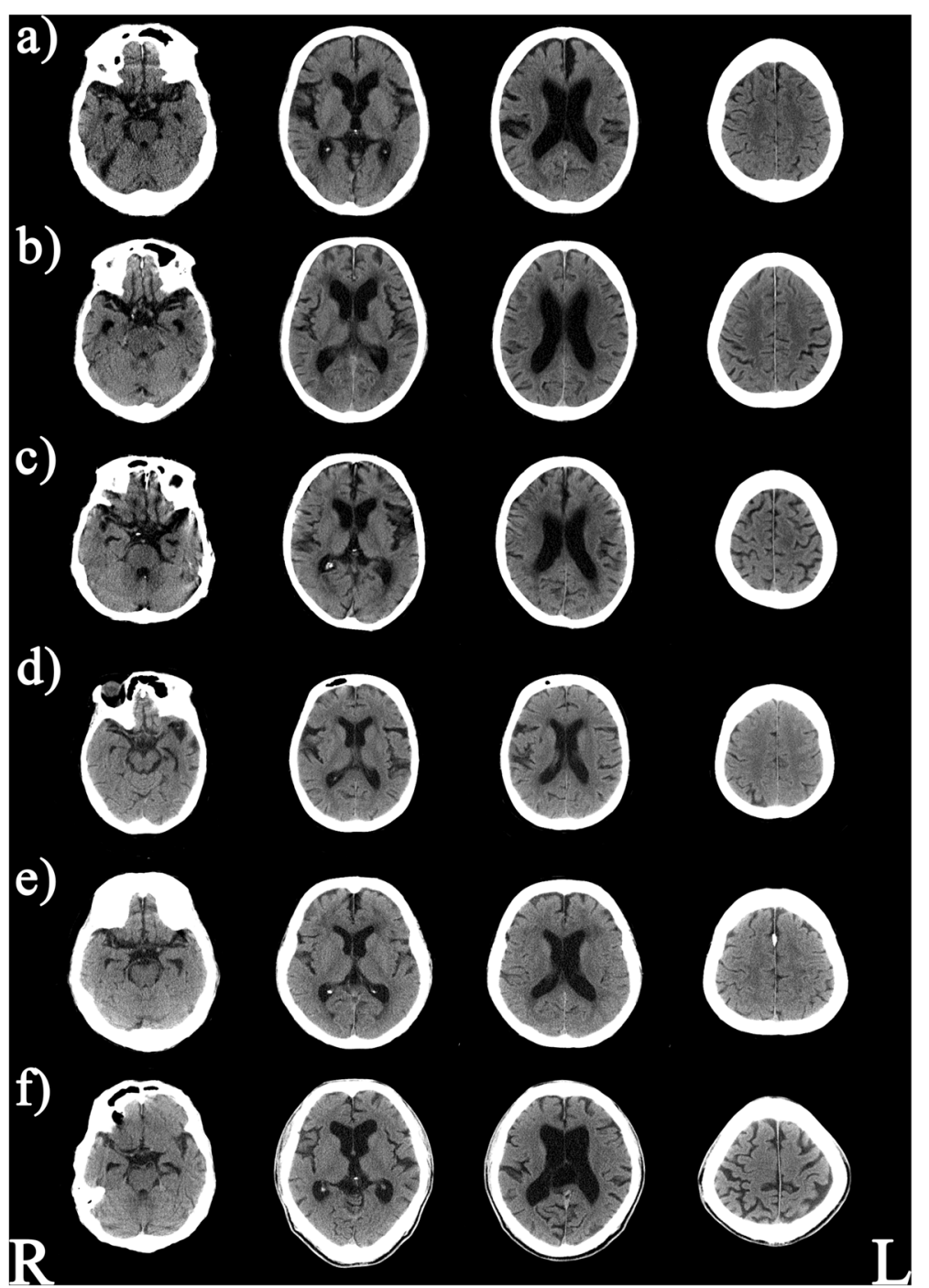

Fig. 1 Shown are computed tomography images of $\mathbf{a}$ an 84-year-old male; $\mathbf{b}$ a 78-year-old male; $\mathbf{c}$ a 74-year-old male; $\mathbf{d}$ a 64-year-old female; and $\mathbf{e}$ a 61-year-old male with idiopathic normal pressure hydrocephalus. Panel $\mathbf{f}$ shows a computed tomography image of a 62-year-old male with secondary normal pressure hydrocephalus with a mass in the lateral ventricle. All subjects have features of an enlarged Sylvian fissure associated with ventriculomegaly and compression of high convexity. Several subjects $(\mathbf{a}, \mathbf{b}, \mathbf{d}$, and $\mathbf{e})$ have disproportionately enlarged subarachnoid space hydrocephalus findings

(14.3\%) [28]. The total rate from the combined data was eight of 59 (13.6\%), which is approximately 4-20 times higher than that of the Japanese general population (0.5-2.9\%) [27, 40, 41]. Consistently, Vanhala et al. [5] estimated that SZ patients were present 3 times more frequently among Finnish iNPH patients compared to the general aged population. However, this prevalence was estimated from already diagnosed iNPH patients. SZ patients hesitate to visit not only psychiatrists but also doctors for physical problems for several reasons. One reason is that they do not recognize physical symptoms due to the lack of feeling unpleasant stimulation such as pain [42]. The second reason is that barriers to receiving health care exist such as a lack of access to health care, lack of integration of medical and mental health systems, and denial of illness [43]. Indeed, several patients with possible iNPH denied undergoing a CSF tap test after informing them of the diagnosis and how to treat the illness. In some cases, we could not determine a diagnosis of possible iNPH due to the patient's cognitive dysfunction, inability to access the hospital for treatment, and their prognosis. In the future, we must find a way that allows SZ patients with possible iNPH to easily receive the required medical care. For these reasons, we think that the actual prevalence of iNPH in SZ patients is higher than estimated by Vanhala et al. [5].

Second, we found significant changes in CT findings of DESH in addition to Evans' index between the group 
Table 2 Demographic and clinical data of patients with possible iNPH and without iNPH (no iNPH) with combined data (present and preceding study)

\begin{tabular}{|c|c|c|c|}
\hline Characteristics & No iNPH $(n=51)$ & Possible iNPH $(n=8)$ & $p$ value \\
\hline Sex, male & 29 & 6 & 1.0 \\
\hline Age (years) & $69.0 \pm 6.8$ & $74.0 \pm 8.5$ & 0.092 \\
\hline $\mathrm{BMI}\left(\mathrm{kg} / \mathrm{m}^{2}\right)$ & $20.5 \pm 2.5(46)$ & $21.9 \pm 2.9(7)$ & 0.294 \\
\hline Smoking & 13 & 0 & 0.176 \\
\hline Age of onset (years) & $27.1 \pm 12.9$ & $25.0 \pm 7.9$ & 0.912 \\
\hline Duration of illness (months) & $41.6 \pm 11.3$ & $53.9 \pm 21.3$ & 0.069 \\
\hline $\mathrm{CP}$ equivalent & $717.0 \pm 493.5$ & $655.4 \pm 497.0$ & 0.842 \\
\hline \multicolumn{4}{|l|}{ Drug administration } \\
\hline Anticholinergics & 20 & 2 & 0.698 \\
\hline BPRS & $41.8 \pm 13.2$ & $43.3 \pm 13.4$ & 0.765 \\
\hline DIEPSS & $5.9 \pm 4.5$ & $10.9 \pm 4.2$ & 0.006 \\
\hline \multicolumn{4}{|l|}{ iNPHGS } \\
\hline GS-Gait & $0.96 \pm 1.1$ & $2.3 \pm 1.5$ & 0.015 \\
\hline GS-Cogn & $1.8 \pm 1.2$ & $2.5 \pm 1.2$ & 0.107 \\
\hline GS-Urin & $0.96 \pm 1.1$ & $1.8 \pm 1.7$ & 0.178 \\
\hline TUG & $16.4 \pm 9.0(44)$ & $24.2 \pm 14.9(6)$ & 0.376 \\
\hline 10-m walking test & $15.2 \pm 6.1(44)$ & $25.2 \pm 14.2(6)$ & 0.064 \\
\hline GSS & $3.1 \pm 3.2(47)$ & $7.7 \pm 4.8(6)$ & 0.009 \\
\hline MMSE & $18.9 \pm 5.5(48)$ & $16.1 \pm 7.2(7)$ & 0.317 \\
\hline NPI & $15.8 \pm 11.4$ & $16.6 \pm 22.3$ & 0.451 \\
\hline Evans' Index & $0.28 \pm 0.04$ & $0.37 \pm 0.07$ & $<0.001$ \\
\hline DESH & 4 & 5 & 0.001 \\
\hline APOE ع4+/ع4- & $11 / 40$ & $2 / 6$ & 1.0 \\
\hline
\end{tabular}

Values denote number (\%) or mean \pm standard deviation

$B M I$ body mass index, CP equivalent chlorpromazine equivalent, BPRS Brief Psychiatric Rating Scale, DIEPSS Drug-Induced Extra-pyramidal Symptoms Scale, iNPH idiopathic normal pressure hydrocephalus, iNPHGS idiopathic Normal-Pressure Hydrocephalus Grading Scale, GS-Gait iNPHGS for gait, GS-Cogn iNPHGS for cognition, GS-Urin iNPHGS for urinary function, TUG Timed Up-and-Go test, GSS Gait Status Scale, MMSE Mini-Mental State Examination, NPI neuropsychiatric inventory

Table 3 Subscales of the Drug-Induced Extra-pyramidal Symptoms Scale (DIEPSS)

\begin{tabular}{llll}
\hline Items & No iNPH $(n=51)$ & Possible iNPH $(n=8)$ & $p$ value \\
\hline Gait & $1.0 \pm 1.4$ & $2.0 \pm 1.9$ & 0.021 \\
Bradykinesia & $1.2 \pm 1.1$ & $1.8 \pm 0.8$ & 0.015 \\
Sialorrhea & $0.6 \pm 0.9$ & $0.4 \pm 0.5$ & 0.531 \\
Rigidity & $0.6 \pm 0.9$ & $1.6 \pm 1.5$ & 0.063 \\
Tremor & $0.7 \pm 1.0$ & $1.2 \pm 1.6$ & 0.376 \\
Akathisia & $0.1 \pm 0.4$ & $0.6 \pm 0.5$ & 0.021 \\
Dystonia & $0.1 \pm 0.3$ & $0.4 \pm 0.5$ & 0.145 \\
Dyskinesia & $0.2 \pm 0.7$ & $0.6 \pm 0.9$ & 0.468 \\
Global & $1.2 \pm 0.9$ & $1.8 \pm 0.4$ & 0.011 \\
\hline
\end{tabular}

The average differences in the DIEPSS subscales were analyzed with the Student's $t$-test or Mann-Whitney $\mathrm{U}$ test with Bonferroni correction (statistical significance: $p<0.006$ )
Table 4 Subscales of the Mini-Mental State Examination (MMSE)

\begin{tabular}{llll}
\hline Items & $\begin{array}{l}\text { No iNPH } \\
(n=48 / 51)\end{array}$ & $\begin{array}{l}\text { Possible iNPH } \\
(n=7 / 8)\end{array}$ & $P$ value \\
\hline Orientation in time & $3.3 \pm 1.6$ & $1.6 \pm 1.3$ & 0.012 \\
Orientation in place & $3.7 \pm 1.3$ & $3.7 \pm 1.5$ & 0.968 \\
Registration & $2.9 \pm 0.4$ & $2.3 \pm 1.3$ & 0.048 \\
Serial-7 & $1.1 \pm 1.4$ & $1.7 \pm 1.6$ & 0.124 \\
Repetition & $0.9 \pm 0.3$ & $0.7 \pm 0.5$ & 0.189 \\
Three-stage command & $2.1 \pm 1.0$ & $2.3 \pm 1.0$ & 0.724 \\
Reading & $0.8 \pm 0.4$ & $0.9 \pm 0.4$ & 0.179 \\
Recall & $1.2 \pm 1.1$ & $0.9 \pm 0.9$ & 0.443 \\
Naming & $2.0 \pm 0.2$ & $1.7 \pm 0.5$ & 0.023 \\
Writing & $0.6 \pm 0.5$ & $0.3 \pm 0.5$ & 0.179 \\
Construction (cube-copying) & $0.3 \pm 0.5$ & $0.1 \pm 0.4$ & 0.423 \\
\hline
\end{tabular}

The average differences in the MMSE subscales were analyzed with the Student's $t$-test or Mann-Whitney $\mathrm{U}$ test with Bonferroni correction (statistical significance: $p<0.0045$ ) 
with possible iNPH and the group with no iNPH. An Evans' index greater than 0.3 is one of the required diagnosis criteria [29], but an Evans' index greater than 0.3 was found in some SZ patients without iNPH. The Evans' index generally reflects ventriculomegaly [44]. However, enlargement of the lateral ventricles occurs with nonspecific global brain atrophy associated with aging even in healthy subjects and those with neurodegeneration [45]. Patients with chronic SZ also have overall brain atrophy and enlargement of ventricular volumes [46]. We should pay more attention to the use of the Evans' index for diagnosis of iNPH in SZ patients. DESH is a reliable marker of diagnosis $[47,48]$ and shunt responsiveness [49]. In addition, several reports also indicate the usefulness of the callosal angle for diagnostic [50, 51] and shunt responsiveness [52-54]. We think that using DESH findings in addition to the Evans' index for making a diagnosis of $\mathrm{iNPH}$ in SZ patients is better than using the Evans' index only.

Third, we explored useful examination values for diagnosis of iNPH by considering three main symptoms, which are dementia, gait disturbance, and urinary incontinence $[9,10]$. In terms of dementia, we found no significant difference in MMSE or iNPHGS-Cogn between the iNPH and no iNPH groups. The progressive cognitive dysfunction in SZ may affect these results [55]. Moreover, the main purpose of MMSE is to understand general cognitive dysfunction. Especially in iNPH cognitive dysfunction, psychomotor slowing, executive dysfunction, and apathy are well-known symptoms $[35,56]$, and these symptoms are typically characterized as frontal lobe impairment [57]. These findings are consistent with single photon emission computed tomography and positron emission tomography studies $[58,59]$. As the previous study showed [60], the use of frontal cognitive assessments such as word fluency, Trail Making Test A, and the Frontal Assessment Battery for understanding the cognitive dysfunction in SZ with iNPH patients is better than MMSE. In terms of side effect of drugs, antipsychotics could induce cognitive dysfunction as a side effect. Especially, clinicians should pay attention to SZ patients who take antipsychotics that have an anticholinergic effect [61, 62]. In addition, it has been reported that the tapering of benzodiazepine and anticholinergic rescue cognitive function [63, 64]. Regarding urinary incontinence, iNPHGS-Urin was not significantly changed in the iNPH group compared to the no iNPH group. The risk of urinary incontinence in SZ patients is 1.78fold higher than that in non-SZ patients [65]. Even here, it has been reported that one of the reasons for urinary dysfunction including urinary inconsistent and retention is the anticholinergic effect in antipsychotics [66]. The fact that urinary incontinence occurred during SZ treatment may possibly mask urinary incontinence as an
iNPH symptom. From the viewpoint of gait disturbance, we found significant changes in the total score of DIEPSS, iNPHGS-Gait, and GSS by analyzing combined data. Here, our question is how we can distinguish gait disturbance that is part of iNPH from that caused by side effects of antipsychotics. The most common mechanism of drug-induced parkinsonism is the blockade of D2 receptor in the striatum. To examine that point, we additionally analyzed the subscales of DIEPSS. We found that subscales of gait and bradykinesia tended to increase in the iNPH with SZ group even though the difference did not reach statistical significance after Bonferroni correction. We consider that SZ patients with a high score for gait and bradykinesia and a low score for other parameters in DIEPSS should be suspected as having iNPH. Furthermore, older patients generally have decreased liver and kidney function, and they easy to get the side effects of antipsychotics [67]. To review the antipsychotics prescription toward older SZ patients will help us to distinguish the gait disturbance of iNPH symptoms from that of side effect.

In addition, we would like to mention the suspected overlapping pathogenesis between SZ and iNPH even though this will be speculative. One autopsy study revealed a high overlapping rate $(8 / 9,89 \%)$ between iNPH and $\mathrm{AD}$ [68]. To examine this point, we did the genotyping for $A P O E$ and unable to find the difference of $\varepsilon 4+$ frequency between iNPH with SZ and iNPH without SZ group. However, $\varepsilon 4+$ frequency was higher regardless of iNPH in this study (non-iNPH vs iNPH $=32 \%$ vs $25 \%$ ) than that of Japanese SZ population (11.1-18.3\%) [6971]. We think that it is hard to conclude this point due to the small sample size of this study. In terms of CSF marker, elevated IL- 6 and -8 were reported in patients with iNPH $[72,73]$. These findings are the same as SZ population as reviewed in [74]. These elevated cytokines are thought to reflect the inflammatory changes of iNPH pathogenesis in the brain. As mentioned in introduction section, vascular etiology is the consistent risk factor for developing iNPH. Among SZ patients, metabolic syndrome is a common comorbidity [2], which may be the one reason to indicate the high prevalence of iNPH in SZ population.

Our study has a few limitations. First, two of three possible iNPH patients declined to undergo the gait disturbance examinations (TUG, 10-m walking test, and GSS). This may have affected our observation of no significant difference in examinations between the no iNPH and possible iNPH patients in this study, even though our preceding study showed significant differences in the TUG and $10-\mathrm{m}$ walking test ${ }^{20}$. Second, selection bias may have been present in this study because we recruited only inpatients. That is extremely limited SZ population, which is relatively far from general SZ 
population. Further studies with larger samples including outpatients are needed to reach a conclusion about the prevalence of iNPH and to reveal which examination is useful for diagnosis. Finally, we could not completely exclude other comorbid conditions, especially AD. Several studies have reported that patients with NPH had a high comorbid ratio of neuropathological change of $\mathrm{AD}$ by conducting biopsy at the time of shunt surgery $(7 / 21$ [33\%], [75]; 23/55 [42\%], [76]).

\section{Conclusions}

The prevalence of iNPH may be higher in older SZ patients than in the general population. We should pay more attention to the three main symptoms of iNPH when we treat older SZ patients. The DIEPSS subscales of gait and bradykinesia and DESH findings may be useful for diagnosing possible iNPH in SZ patients. Psychiatrists are responsible for diagnosing and treating iNPH in older SZ patients without overlooking this possibility. In daily medical care of SZ patients, clinicians possibly could find the early stage of iNPH by paying attention to those symptoms and provide the early treatment for iNPH patients in the older SZ population. To verify the iNPH symptoms clearly in older SZ patients, we had better to decrease benzodiazepine and anticholinergics, and optimize the doses of antipsychotics.

\section{Abbreviations}

AD: Alzheimer's Disease; APOE: Apolipoprotein E; BPRS: Brief Psychiatric Rating Scale; CT: Computed Tomography; DIEPSS: Drug-Induced Extrapyramidal Symptoms Scale; DSM-5: Diagnosis and Statistical Manual of Mental Disorder; GSS: Gait Status Scale; iNPH: idiopathic Normal Pressure Hydrocephalus; iNPHGS: iNPH Grading Scale; MMSE: Mini-Mental State Examination; NPI: NeuroPsychiatric Inventory; PANSS: Positive And Negative Symptom Scale; SZ: Schizophrenia; TUG: Timed Up and Go

\section{Acknowledgments}

We wish to thank Ms. Chiemi Onishi for technical assistance and Dr. Norio Kuroda and Dr. Taisei Kuroda for understanding and agreeing with this study. We would like to thank the staff of Matsukaze Hospital, Juzen Yurinoki Hospital, Shokokai Imabari Hospital, and Kuroda Hospital, Ehime, Japan for understanding the purpose of this study.

\section{Authors' contributions}

YY conceptualized, generated, analyzed the data, and wrote manuscript. TY analyzed data and co-wrote manuscript. HM, MN, MA, HO, SI, YM, YT, NY, NT, MM, YO, and SO collected data. JI interpreted the data and reviewed manuscript. SU edited the manuscript. All authors read and approved the final manuscript.

\section{Funding}

This work was partially supported by a Health and Labour Science Research Grant from the Japanese Ministry of Health, Labour and Welfare, and a Grant-in-Aid for Scientific Research from the Japanese Ministry of Education, Culture, Sports, Science and Technology, JSPS KAKENHI Grant Numbers 17 K16381 and $18 \mathrm{~K} 07564$. These funders had no role in the study design; data collection, analysis and interpretation of data; in writing the manuscript; and in the decision to submit the paper for publication.

\section{Availability of data and materials}

The datasets used and/or analyzed during the current study are available from the corresponding author on reasonable request.

\section{Ethics approval and consent to participate}

Ethical approval has been obtained for the study from the institutional ethics committees of Matsukaze Hospital, Juzen Yurinoki Hospital, Shokokai Imabari Hospital, and Ehime University Graduate School of Medicine. Informed consent was obtained in writing from all participants.

\section{Consent for publication}

Not applicable.

\section{Competing interests}

All of authors have no competing interests.

\section{Author details}

'Department of Neuropsychiatry, Molecules and Function, Ehime University Graduate School of Medicine, Shitsukawa, Toon, Ehime 791-0295, Japan. ${ }^{2}$ Department of Psychiatry, Matsukaze Hospital, Doicho Irino 970, Shikokutyuo, Ehime, Japan. ${ }^{3}$ Department of Psychiatry, Juzen Yurinoki Hospital, Sumino Nittacho 1-1-28, Niihama, Ehime, Japan. ${ }^{4}$ Department of Neuropsychiatry, Shokokai Imabari Hospital, Takaichi 786-13, Imabari, Ehime, Japan.

Received: 27 February 2020 Accepted: 20 May 2020

Published online: 01 June 2020

\section{References}

1. Carney CP, Jones L, Woolson RF. Medical comorbidity in women and men with schizophrenia: a population-based controlled study. J Gen Intern Med. 2006;21(11):1133-7.

2. Mitchell AJ, Vancampfort D, Sweers $K$, van Winkel R, Yu W, De Hert M. Prevalence of metabolic syndrome and metabolic abnormalities in schizophrenia and related disorders--a systematic review and meta-analysis. Schizophr Bull. 2013;39(2):306-18.

3. De Hert M, Schreurs V, Sweers K, Van Eyck D, Hanssens L, Sinko S, Wampers M, Scheen A, Peuskens J, van Winkel R. Typical and atypical antipsychotics differentially affect long-term incidence rates of the metabolic syndrome in first-episode patients with schizophrenia: a retrospective chart review. Schizophr Res. 2008;101(1-3):295-303.

4. Velligan DI, Weiden PJ, Sajatovic M, Scott J, Carpenter D, Ross R, Docherty JP. Expert consensus panel on adherence problems in S, persistent mental I: The expert consensus guideline series: adherence problems in patients with serious and persistent mental illness. J Clin Psychiatry. 2009;70(Suppl 4):1-46 quiz 47-48.

5. Vanhala V, Junkkari A, Korhonen VE, Kurki MI, Hiltunen M, Rauramaa T, Nerg $\mathrm{O}$, Koivisto AM, Remes AM, Perala J, et al. Prevalence of schizophrenia in idiopathic Normal pressure hydrocephalus. Neurosurgery. 2019;84(4):883-9.

6. Agrawal A, Tiwari AM, Tiple P, Chauhan MK, Nagarale M. Normal pressure hydrocephalus in a case of schizophrenia. Indian J Psychiatry. 2012;54(4): 385-6.

7. Mishra BR, Sarkar S, Mishra S, Praharaj SK, Mahapatra P, Sinha VK. Antipsychotic sensitivity in normal pressure hydrocephalus. Gen Hosp Psychiatry. 2011;33(1):83 e11-83.

8. Maurizi CP. The pathophysiology of enlarged ventricles in normal pressure communicating hydrocephalus and schizophrenia: a possible therapeutic role for melatonin. Med Hypotheses. 1987;23(1):61-6.

9. Hakim S, Adams RD. The special clinical problem of symptomatic hydrocephalus with normal cerebrospinal fluid pressure. Observations on cerebrospinal fluid hydrodynamics. J Neurol Sci. 1965;2(4):307-27.

10. Shprecher D, Schwalb J, Kurlan R. Normal pressure hydrocephalus: diagnosis and treatment. Curr Neurol Neurosci Rep. 2008;8(5):371-6.

11. Green MF. What are the functional consequences of neurocognitive deficits in schizophrenia? Am J Psychiatry. 1996;153(3):321-30.

12. Heinrichs RW, Zakzanis KK. Neurocognitive deficit in schizophrenia: a quantitative review of the evidence. Neuropsychology. 1998;12(3):426-45.

13. Ishikawa M, Hashimoto M, Mori E, Kuwana N, Kazui H. The value of the cerebrospinal fluid tap test for predicting shunt effectiveness in idiopathic normal pressure hydrocephalus. Fluids Barriers CNS. 2012;9(1):1.

14. Ishikawa M, Yamada S, Yamamoto K. Early and delayed assessments of quantitative gait measures to improve the tap test as a predictor of shunt effectiveness in idiopathic normal pressure hydrocephalus. Fluids Barriers CNS. 2016;13(1):20. 
15. Wikkelso C, Andersson H, Blomstrand C, Lindqvist G. The clinical effect of lumbar puncture in normal pressure hydrocephalus. J Neurol Neurosurg Psychiatry. 1982:45(1):64-9.

16. Picascia M, Minafra B, Zangaglia R, Gracardi L, Pozzi NG, Sinforiani E, Pacchetti C. Spectrum of cognitive disorders in idiopathic normal pressure hydrocephalus. Funct Neurol. 2016;31(3):143-7.

17. Junkkari A, Hayrinen A, Rauramaa T, Sintonen H, Nerg O, Koivisto AM, Roine RP, Viinamaki H, Soininen $H$, Luikku A, et al. Health-related quality-of-life outcome in patients with idiopathic normal-pressure hydrocephalus - a 1year follow-up study. Eur J Neurol. 2017;24(1):58-66.

18. Brautigam K, Vakis A, Tsitsipanis C. Pathogenesis of idiopathic Normal pressure hydrocephalus: a review of knowledge. J Clin Neurosci. 2019;61:10 3.

19. Shepherd AM, Laurens KR, Matheson SL, Carr VJ, Green MJ. Systematic meta-review and quality assessment of the structural brain alterations in schizophrenia. Neurosci Biobehav Rev. 2012;36(4):1342-56.

20. Huhtaniska S, Jaaskelainen E, Heikka T, Moilanen JS, Lehtiniemi H, Tohka J, Manjon JV, Coupe P, Bjornholm L, Koponen H, et al. Long-term antipsychotic and benzodiazepine use and brain volume changes in schizophrenia: the northern Finland birth cohort 1966 study. Psychiatry Res Neuroimaging. 2017;266:73-82.

21. Jaraj D, Agerskov S, Rabiei K, Marlow T, Jensen C, Guo X, Kern S, Wikkelso C, Skoog I. Vascular factors in suspected normal pressure hydrocephalus: a population-based study. Neurology. 2016;86(7):592-9.

22. Israelsson H, Carlberg B, Wikkelso C, Laurell K, Kahlon B, Leijon G, Eklund A Malm J. Vascular risk factors in INPH: a prospective case-control study (the INPH-CRasH study). Neurology. 2017;88(6):577-85.

23. Suvisaari J, Keinanen J, Eskelinen S, Mantere O. Diabetes and schizophrenia. Curr Diab Rep. 2016;16(2):16.

24. Casmiro M, Benassi G, Cacciatore FM, D'Alessandro R. Frequency of idiopathic normal pressure hydrocephalus. Arch Neurol. 1989;46(6):608.

25. Graff-Radford NR, Godersky JC. Idiopathic normal pressure hydrocephalus and systemic hypertension. Neurology. 1987;37(5):868-71.

26. Krauss JK, Regel JP, Vach W, Droste DW, Borremans JJ, Mergner T. Vascular risk factors and arteriosclerotic disease in idiopathic normal-pressure hydrocephalus of the elderly. Stroke. 1996;27(1):24-9.

27. Iseki C, Kawanami T, Nagasawa H, Wada M, Koyama S, Kikuchi K, Arawaka S, Kurita K, Daimon M, Mori E, et al. Asymptomatic ventriculomegaly with features of idiopathic normal pressure hydrocephalus on MRI (AVIM) in the elderly: a prospective study in a Japanese population. J Neurol Sci. 2009; 277(1-2):54-7.

28. Yoshino Y, Yoshida T, Mori T, Hirota S, Iga J, Ueno S. Risk of idiopathic normal pressure hydrocephalus in older inpatients with schizophrenia. Int Psychogeriatr. 2016;28(5):863-8

29. Mori E, Ishikawa M, Kato T, Kazui H, Miyake H, Miyajima M, Nakajima M, Hashimoto M, Kuriyama N, Tokuda T, et al. Guidelines for management of idiopathic normal pressure hydrocephalus: second edition. Neurol Med Chir (Tokyo). 2012;52(11):775-809.

30. Kay SR, Fiszbein A, Opler LA. The positive and negative syndrome scale (PANSS) for schizophrenia. Schizophr Bull. 1987;13(2):261-76.

31. Leucht S, Rothe P, Davis JM, Engel RR. Equipercentile linking of the BPRS and the PANSS. Eur Neuropsychopharmacol. 2013;23(8):956-9.

32. Inada T. DIEPSS : a second generation rating scale for antipsychotic induced extrapyramidal symptoms Drug induced Extrapyramidal Symptoms Scale. Tokyo: Seiwa Shoten; 2009.

33. Kubo Y, Kazui H, Yoshida T, Kito Y, Kimura N, Tokunaga H, Ogino A, Miyake H, Ishikawa M, Takeda M. Validation of grading scale for evaluating symptoms of idiopathic normal-pressure hydrocephalus. Dement Geriatr Cogn Disord. 2008;25(1):37-45.

34. Podsiadlo D, Richardson S. The timed "up \& go": a test of basic functional mobility for frail elderly persons. J Am Geriatr Soc. 1991;39(2):142-8.

35. Miyoshi N, Kazui H, Ogino A, Ishikawa M, Miyake $H$, Tokunaga $H$, Ikejiri $Y$, Takeda M. Association between cognitive impairment and gait disturbance in patients with idiopathic normal pressure hydrocephalus. Dement Geriatr Cogn Disord. 2005;20(2-3):71-6.

36. Folstein MF, Folstein SE, McHugh PR. "mini-mental state". A practical method for grading the cognitive state of patients for the clinician. J Psychiatr Res. 1975;12(3):189-98.

37. Cummings JL, Mega M, Gray K, Rosenberg-Thompson S, Carusi DA, Gornbein J. The neuropsychiatric inventory: comprehensive assessment of psychopathology in dementia. Neurology. 1994;44(12):2308-14.
38. Guo H, Tabara Y, Igase M, Yamamoto M, Ochi N, Kido T, Uetani E, Taguchi K, Miki T, Kohara K. Abnormal nocturnal blood pressure profile is associated with mild cognitive impairment in the elderly: the J-SHIPP study. Hypertens Res. 2010;33(1):32-6.

39. Kanda Y. Investigation of the freely available easy-to-use software 'EZR' for medical statistics. Bone Marrow Transplant. 2013:48(3):452-8.

40. Hiraoka K, Meguro K, Mori E. Prevalence of idiopathic normal-pressure hydrocephalus in the elderly population of a Japanese rural community. Neurol Med Chir (Tokyo). 2008;48(5):197-9 discussion 199-200.

41. Tanaka N, Yamaguchi S, Ishikawa H, Ishii H, Meguro K. Prevalence of possible idiopathic normal-pressure hydrocephalus in Japan: the Osaki-Tajiri project. Neuroepidemiology. 2009;32(3):171-5.

42. Dworkin RH. Pain insensitivity in schizophrenia: a neglected phenomenon and some implications. Schizophr Bull. 1994;20(2):235-48.

43. Goldman LS. Medical illness in patients with schizophrenia. J Clin Psychiatry. 1999;60(Suppl 21):10-5.

44. Raftopoulos C, Deleval J, Chaskis C, Leonard A, Cantraine F, Desmyttere F, Clarysse S, Brotchi J. Cognitive recovery in idiopathic normal pressure hydrocephalus: a prospective study. Neurosurgery. 1994;35(3):397-404 discussion 404-395.

45. Apostolova LG, Green AE, Babakchanian S, Hwang KS, Chou YY, Toga AW Thompson PM. Hippocampal atrophy and ventricular enlargement in normal aging, mild cognitive impairment $(\mathrm{MCl})$, and Alzheimer disease. Alzheimer Dis Assoc Disord. 2012;26(1):17-27.

46. DeLisi LE, Sakuma M, Tew W, Kushner M, Hoff AL, Grimson R. Schizophrenia as a chronic active brain process: a study of progressive brain structural change subsequent to the onset of schizophrenia. Psychiatry Res. 1997; 74(3):129-40.

47. Garcia-Armengol R, Domenech S, Botella-Campos C, Goncalves FJ, Menendez B, Teixidor P, Munoz-Narbona L, Rimbau J. Comparison of elevated intracranial pressure pulse amplitude and disproportionately enlarged subarachnoid space (DESH) for prediction of surgical results in suspected idiopathic normal pressure hydrocephalus. Acta Neurochir. 2016; 158(11):2207-13.

48. Hashimoto M, Ishikawa M, Mori E, Kuwana N. Study of loni: Diagnosis of idiopathic normal pressure hydrocephalus is supported by MRI-based scheme: a prospective cohort study. Cerebrospinal Fluid Res. 2010;7:18.

49. Craven $\mathrm{CL}$, Toma AK, Mostafa $\mathrm{T}$, Patel N, Watkins LD. The predictive value of DESH for shunt responsiveness in idiopathic normal pressure hydrocephalus. J Clin Neurosci. 2016;34:294-8.

50. Cagnin A, Simioni M, Tagliapietra M, Citton V, Pompanin S, Della Puppa A, Ermani M, Manara R. A simplified Callosal angle measure best differentiates idiopathic-Normal pressure hydrocephalus from neurodegenerative dementia. J Alzheimers Dis. 2015:46(4):1033-8.

51. Ishii K, Kanda T, Harada A, Miyamoto N, Kawaguchi T, Shimada K, Ohkawa S, Uemura T, Yoshikawa T, Mori E. Clinical impact of the callosal angle in the diagnosis of idiopathic normal pressure hydrocephalus. Eur Radiol. 2008; 18(11):2678-83.

52. Grahnke K, Jusue-Torres I, Szujewski C, Joyce C, Schneck M, Prabhu VC, Anderson DE. The quest for predicting sustained shunt response in Normalpressure hydrocephalus: an analysis of the Callosal Angle's utility. World Neurosurg. 2018;115:e717-22.

53. Virhammar J, Laurell K, Cesarini KG, Larsson EM. The callosal angle measured on MRI as a predictor of outcome in idiopathic normal-pressure hydrocephalus. J Neurosurg. 2014;120(1):178-84.

54. Virhammar J, Laurell K, Cesarini KG, Larsson EM. Increase in callosal angle and decrease in ventricular volume after shunt surgery in patients with idiopathic normal pressure hydrocephalus. J Neurosurg. 2018;130(1):130-5.

55. Green MF, Kern RS, Heaton RK. Longitudinal studies of cognition and functional outcome in schizophrenia: implications for MATRICS. Schizophr Res. 2004;72(1):41-51.

56. Boon AJ, Tans JT, Delwel EJ, Egeler-Peerdeman SM, Hanlo PW, Wurzer HA, Avezaat CJ, de Jong DA, Gooskens RH, Hermans J. Dutch normal-pressure hydrocephalus study: prediction of outcome after shunting by resistance to outflow of cerebrospinal fluid. J Neurosurg. 1997;87(5):687-93.

57. Ogino A, Kazui H, Miyoshi N, Hashimoto M, Ohkawa S, Tokunaga H, Ikejiri Y, Takeda M. Cognitive impairment in patients with idiopathic normal pressure hydrocephalus. Dement Geriatr Cogn Disord. 2006;21(2):113-9.

58. Kristensen B, Malm J, Fagerland M, Hietala SO, Johansson B, Ekstedt J, Karlsson T. Regional cerebral blood flow, white matter abnormalities, and 
cerebrospinal fluid hydrodynamics in patients with idiopathic adult hydrocephalus syndrome. J Neurol Neurosurg Psychiatry. 1996;60(3):282-8.

59. Momjian S, Owler BK, Czosnyka Z, Czosnyka M, Pena A, Pickard JD. Pattern of white matter regional cerebral blood flow and autoregulation in normal pressure hydrocephalus. Brain. 2004;127(Pt 5):965-72.

60. Saito M, Nishio Y, Kanno S, Uchiyama M, Hayashi A, Takagi M, Kikuchi H, Yamasaki H, Shimomura T, lizuka O, et al. Cognitive profile of idiopathic normal pressure hydrocephalus. Dement Geriatr Cogn Dis Extra. 2011;1(1): 202-11.

61. Kim SJ, Jung D, Shim JC, Moon JJ, Jeon DW, Kim YN, Seo YS, Jung SS, Seo BJ, Kim JE. The effect of anticholinergic burden on cognitive and daily living functions in patients with schizophrenia. Asian J Psychiatr. 2019;46:111-7.

62. Minzenberg MJ, Poole JH, Benton C, Vinogradov S. Association of anticholinergic load with impairment of complex attention and memory in schizophrenia. Am J Psychiatry. 2004;161(1):116-24.

63. Kitajima R, Miyamoto S, Tenjin T, Ojima K, Ogino S, Miyake N, Fujiwara K, Funamoto Y, Arai J, Tsukahara S, et al. Effects of tapering of long-term benzodiazepines on cognitive function in patients with schizophrenia receiving a second-generation antipsychotic. Prog Neuro-Psychopharmacol Biol Psychiatry. 2012;36(2):300-6.

64. Ogino S, Miyamoto S, Miyake N, Yamaguchi N. Benefits and limits of anticholinergic use in schizophrenia: focusing on its effect on cognitive function. Psychiatry Clin Neurosci. 2014;68(1):37-49.

65. Hsu WY, Muo CH, Ma SP, Kao CH. Association between schizophrenia and urinary incontinence: a population-based study. Psychiatry Res. 2017;248:359.

66. Faure Walker N, Brinchmann K, Batura D. Linking the evidence between urinary retention and antipsychotic or antidepressant drugs: a systematic review. Neurourol Urodyn. 2016;35(8):866-74.

67. Gareri P, Segura-Garcia C, Manfredi VG, Bruni A, Ciambrone P, Cerminara G, De Sarro G, De Fazio P. Use of atypical antipsychotics in the elderly: a clinical review. Clin Interv Aging. 2014;9:1363-73.

68. Cabral D, Beach TG, Vedders L, Sue LI, Jacobson S, Myers K, Sabbagh MN. Frequency of Alzheimer's disease pathology at autopsy in patients with clinical normal pressure hydrocephalus. Alzheimers Dement. 2011;7(5):50913.

69. Kimura T, Shono M, Yokota S, Ishizuka K, Watanabe M, Takamatsu J, Miyakawa T. Apolipoprotein E epsilon4 and tardive dyskinesia in a Japanese population. J Psychiatr Res. 2000;34(4-5):329-32

70. Kimura T, Yokota S, Igata-Yi R, Shono M, Takamatsu J, Miyakawa T. Apolipoprotein E epsilon2 allele and early onset schizophrenia. Neurosci Lett. 1997:231(1):53-5.

71. Igata-Yi R, Igata T, Ishizuka K, Kimura T, Sakamoto S, Katsuragi S, Takamatsu J, Miyakawa T. Apolipoprotein E genotype and psychosis. Biol Psychiatry. 1997:41(8):906-8.

72. Czubowicz K, Glowacki M, Fersten E, Kozlowska E, Strosznajder RP, Czernicki Z. Levels of selected pro- and anti-inflammatory cytokines in cerebrospinal fluid in patients with hydrocephalus. Folia Neuropathol. 2017:55(4):301-7.

73. Pyykko OT, Lumela M, Rummukainen J, Nerg O, Seppala TT, Herukka SK, Koivisto AM, Alafuzoff I, Puli L, Savolainen S, et al. Cerebrospinal fluid biomarker and brain biopsy findings in idiopathic normal pressure hydrocephalus. PLoS One. 2014;9(3):e91974.

74. Orlovska-Waast S, Kohler-Forsberg O, Brix SW, Nordentoft M, Kondziella D, Krogh J, Benros ME. Cerebrospinal fluid markers of inflammation and infections in schizophrenia and affective disorders: a systematic review and meta-analysis. Mol Psychiatry. 2019;24(6):869-87.

75. Del Bigio MR, Cardoso ER, Halliday WC. Neuropathological changes in chronic adult hydrocephalus: cortical biopsies and autopsy findings. Can J Neurol Sci. 1997:24(2):121-6.

76. Golomb J, Wisoff J, Miller DC, Boksay I, Kluger A, Weiner H, Salton J, Graves W. Alzheimer's disease comorbidity in normal pressure hydrocephalus: prevalence and shunt response. J Neurol Neurosurg Psychiatry. 2000;68(6): $778-81$

\section{Publisher's Note}

Springer Nature remains neutral with regard to jurisdictional claims in published maps and institutional affiliations.

\section{Ready to submit your research? Choose BMC and benefit from:}

- fast, convenient online submission

- thorough peer review by experienced researchers in your field

- rapid publication on acceptance

- support for research data, including large and complex data types

- gold Open Access which fosters wider collaboration and increased citations

- maximum visibility for your research: over $100 \mathrm{M}$ website views per year

At $\mathrm{BMC}$, research is always in progress.

Learn more biomedcentral.com/submissions 